\title{
Soliton ratchets out of point-like inhomogeneities
}

\author{
L. Morales-Molina ${ }^{1,2}$, F.G. Mertens ${ }^{1}$, and A. Sánchez ${ }^{2, a}$ \\ 1 Physikalisches Institut, Universität Bayreuth, 85440 Bayreuth, Germany \\ 2 Grupo Interdisciplinar de Sistemas Complejos (GISC) and Departamento de Matemáticas, Universidad Carlos III de Madrid, \\ Avenida de la Universidad 30, 28911 Leganés, Madrid, Spain
}

Received 1st July 2003 / Received in final form 25 November 2003

\begin{abstract}
We introduce and study a novel design for a ratchet potential for soliton excitations. The potential is implemented by means of an array of point-like (delta) inhomogeneities in an otherwise homogeneous potential. We use collective coordinates which predict that the effective potential acting on the soliton is periodic but asymmetric and gives rise to the ratchet effect. Numerical simulations fully confirm this prediction; quantitative agreement is reached by an improved version of the theory. Although we specifically show that it is most interesting for building Josephson junction ratchets capable to rectify time-symmetric ac forces, the proposed mechanism is very general and can appear in many contexts, including biological systems.
\end{abstract}

PACS. 05.45.Yv Solitons - 05.60.-k Transport processes - 63.20.Pw Localized modes

\section{Introduction}

Ratchet systems have been the object of intensive studies due to their promising applications in biological systems $[1,2]$ and micro- and nano-technologies [3,4]. Recently, a great deal of effort has been devoted to generalize the ratchet mechanism for point particles to spatially extended systems [5]. One proposal along this direction relates to the existence of net transport in homogeneous extended systems driven by homogeneous ac forces $[6,7]$. However, although this is a very good and feasible way to induce transport with specified properties, it cannot be used to rectify time-symmetric forces, because it relies on the breaking of the time symmetry of the ac force. In case the ac force is symmetric, the alternative route to rectification is to introduce spatial inhomogeneity. Models in this class have been studied theoretically [8] and also implemented in superconducting devices $[4,9]$. In this case, a drawback is the difficulty of their fabrication at the microor nano-scale, because controlling the asymmetry is very complex [4]. An additional factor that has to be considered in this context is the interplay between disorder and nonlinearity, which can be of fundamental relevance in the design of these new transport devices and particularly when competition of scales takes place $[10,11]$.

In this letter, we present a much simpler design for an extended ratchet that works irrespective of the symmetry of the ac force. Specifically, we focus on the sine-Gordon (sG) model, among other reasons because of its important applications to superconducting devices such as long

\footnotetext{
a e-mail: anxo@math.uc3m.es
}

Josephson Junctions (LJJ). In this context, our proposal is based on the inclusion of point-like inhomogeneities, which correspond to micro-shorts along the LJJ $[12,13]$. Notwithstanding, the mechanism is very general and it can be applied to many other soliton-bearing systems where the interaction of kinks with point-like inhomogeneities is similar to that occurring in the sG model [14-16]. We begin by introducing the perturbed sG model, driven by an ac force in the presence of a periodic array of inhomogeneities. We first study analytically this problem by means of a collective coordinate for the motion of the kink center. We thus show that under certain conditions the effective kink dynamics is that of a particle ratchet. We then study, by numerical simulations of the full system, the behavior of the mean velocity as a function of the driving amplitude for different frequencies. Although qualitative agreement with the analytical predictions is found, the quantitative comparison is not so good. We subsequently improve our theory by introducing the width degree of freedom, whose quantitative success makes clear the physical mechanism needed for a correct description of the phenomenon. We conclude by summarizing our main results and pointing out future research along these lines.

\section{Model}

Our starting point is the following perturbed equation:

$\phi_{t t}+\beta \phi_{t}-\phi_{x x}+[1+V(x)] \sin \phi=A \sin \left(\omega t+\delta_{0}\right)$,

where the term $V(x) \sin (\phi)$ accounts for local inhomogeneities which can be, for instance, variations of the 
critical current in a LJJ, and $A \sin \left(\omega t+\delta_{0}\right) \equiv f(t)$ corresponds to an ac bias current [17]. The kink solution of equation (1) in the absence of perturbations, i.e., $\beta=V=A=0$, is given by the expression

$$
\phi(x, t)=4 \arctan \left(\exp \left[\frac{x-X(t)}{l_{0} \sqrt{1-v^{2}}}\right]\right)
$$

where $X(t)$ and $v=\dot{X}(t)$ are the kink position and velocity, respectively, and $l_{0}$ is a measure of the kink width at rest. For the sine-Gordon case $l_{0}=1$, but we choose to leave this as an explicit parameter to exhibit the physical relevance of this quantity; besides, in many other solitonbearing systems collective coordinate equations have the same form as in this case but $l_{0} \neq 1$ (e.g., the $\phi^{4}$ system). The solution in equation (2) represents, always in the context of LJJ, a flux quantum (fluxon) propagating along the junction.

The question now is: What is the ideal shape of the function $V(x)$ to turn our system into a ratchet device based on the fluxon? It is important to recall that, when driven by symmetric ac forces, a damped fluxon can only exhibit oscillatory motion [19]. This problem was overcome in previous works, and soliton ratchet behavior has been found for this system when the potential of the unperturbed sine-Gordon equation becomes asymmetric [18], or when the system is under an inhomogeneous magnetic field [17]. To our knowledge, none of the previous works resorted to inhomogeneities for breaking the symmetry of the system, as we now do. Our proposal consists of an array of point-like inhomogeneities, which in the case of LJJ can be modelled as delta functions if their length is less than the Josephson penetration length. We will choose $\left\{x_{n}\right\}$ to form a periodic, asymmetric array; in particular, for this work we have specifically chosen three inhomogeneities per spatial period,

$$
\begin{aligned}
V(x)=\epsilon \sum_{n}\left[\delta\left(x-x_{1}-n L\right)+\delta\right. & \left(x-x_{2}-n L\right) \\
& \left.+\delta\left(x-x_{3}-n L\right)\right],
\end{aligned}
$$

where the parameters should satisfy the constraints $a, b, c \sim l_{0} ; a, b<c$ with $a \neq b$, where $L=a+b+c$, $a=x_{2}-x_{1}, b=x_{3}-x_{2}$ and $c=L+x_{1}-x_{3}$, with $x_{1}<x_{2}<x_{3}$. As will be shown below, for a system satisfying these conditions we obtain net motion with a behavior that resembles very closely the one found in ratchetlike systems for point-particles. We have to stress that the distances between the delta functions have to be of the same length scale as the kink width; otherwise, different behaviors could arise like those demonstrated in [15], as the interference between adjacent deltas is lost. Within that requirement, our proposal is very versatile, as it is possible in principle to induce directional motion by using an array whose configuration presents more than three inhomogeneities per period, in case a different ratchet potential were required.

\section{Collective coordinate theory}

As a first step to justify our choice for the perturbative term $V(x)$, we present a simple collective coordinate analysis of its effect on the soliton dynamics. The idea of this well-known approximate technique for treating solitonbearing equations is to assume that perturbations affect mostly the motion of the soliton center (and/or other parameters, as we will see below) and to proceed to a drastic reduction of the number of degrees of freedom by deriving an effective equation for the corresponding collective coordinate (see, e.g., [11] for a recent review and further references). One of the easiest procedures to derive equations for the collective coordinate is by means of the conservations laws, making use of the so-called adiabatic approach, first proposed by McLaughlin and Scott [12]. Following straightforwardly the procedure in this reference, it is a matter of algebra to show, using equation (2) as an Ansatz for the perturbed equation (1), that the corresponding equation of motion for the kink center in the limit of small velocities $v^{2} \ll 1$ is

$$
M_{0} \ddot{X}+\beta M_{0} \dot{X}=-\frac{\partial U}{\partial X}-q A \sin \left(\omega t+\delta_{0}\right),
$$

where

$$
\begin{aligned}
& U(X)=2 \epsilon \sum_{n}\left[\frac{1}{\cosh ^{2}\left(X-x_{1}-n L\right)}\right. \\
& \left.\quad+\frac{1}{\cosh ^{2}\left(X-x_{2}-n L\right)}+\frac{1}{\cosh ^{2}\left(X-x_{3}-n L\right)}\right]
\end{aligned}
$$

is the effective potential function, we have for this one time put $l_{0}=1$, and $M_{0}=8$ and $q=2 \pi$ are the soliton rest mass and topological charge, respectively.

The potential function given by equation (5) is depicted in Figure 1a for the perturbation $V(x)$ defined in equation (3), with $x_{1}=0.5, x_{2}=1 ., x_{3}=2.3$ and period $L=4$. As can be seen from the figure, it corresponds to an asymmetric potential characteristic of ratchet systems; in fact, equation (4) is the same as that for a point particle in a rocking ratchet (see [2]). We stress, however, that equation (4), as it is, describes an inertial rocking ratchet, similar to those studied in [20]. As in this case the dynamics is much more complicated, involving dependencies on the initial conditions and on other factors, in the following we restrict ourselves to the overdamped case (the common situation in ratchet systems [2]) by taking $\beta=1$. As an immediate consequence, within this approximation, we can expect that the soliton center should move towards the left. Figure 1b shows, for one particular value of the frequency, the prediction of equation (4) in this overdamped approach, confirming this expectation and showing the typical window behavior of ratchets (see below).

\section{Numerical results}

To check the prediction of the simple theory we have summarized above, we have carried out numerical simulations of equation (1). We have used a Strauss-Vázquez 

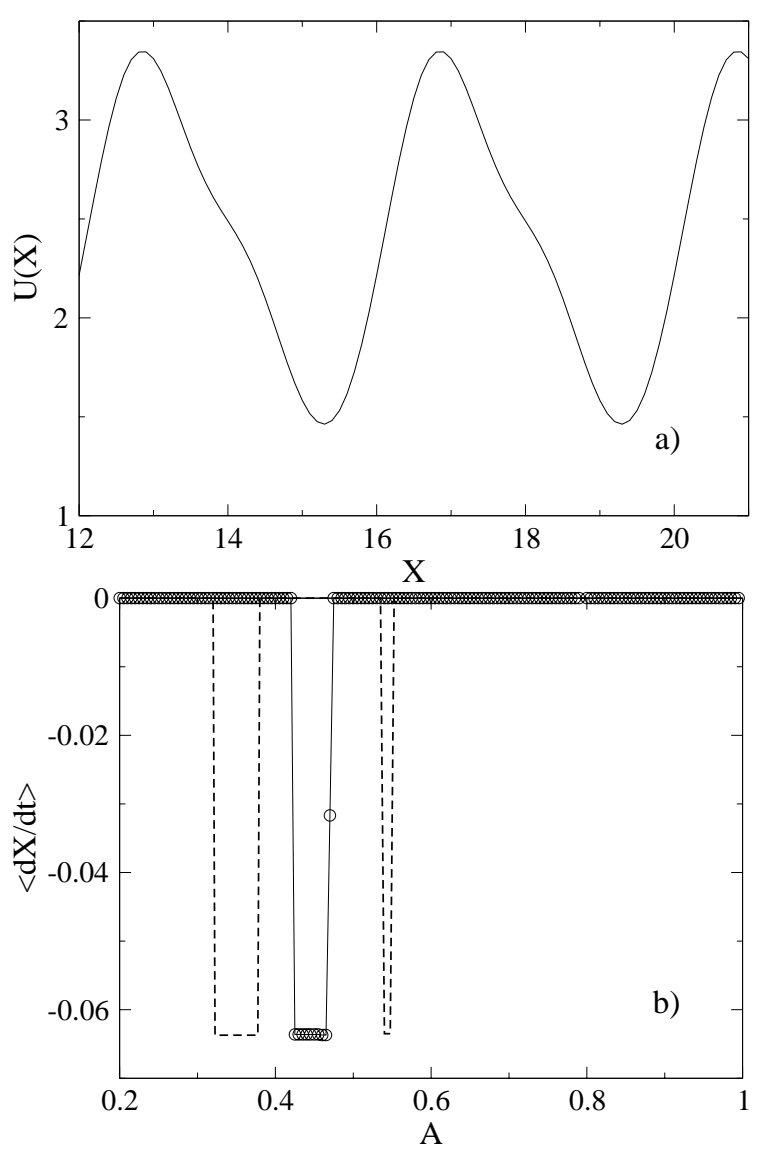

Fig. 1. a) Effective potential for the kink center within the adiabatic approach, originating in the perturbation $V(x)$ defined in equation (3) with $\epsilon=0.8, x_{1}=0.5, x_{2}=1.0, x_{3}=2.3$ and period $L=4$. b) Mean kink velocity $\langle d X / d t\rangle$ vs. driving amplitude $A$ for the frequency $\omega=0.1$. Circles: direct numerical simulation of equation (1), the line being only a guide to the eye; dashed line: collective coordinate approach, equation (4).

numerical scheme [21] with free boundary conditions. The spatial and temporal integration steps were $\Delta x=0.1$ and $\Delta t=0.01$ respectively. The spatial interval for the simulations was $[-50,150]$, with the inhomogeneities arranged periodically according to our three delta unit cell in $[0,100]$. The system was simulated up to times as long as $T=4000$ time units. Finally, the numerical representation for delta functions is $1 / \Delta x$ where the inhomogeneities are located and zero otherwise $[14,15]$.

The simulation results are summarized in Figure 2. As we may see, the system behaves very much like a point particle ratchet (cf., e.g., [22,23]; see also Fig. 5 in [9]). Indeed, we appreciate the existence of windows of motion separated by gaps where the motion is oscillatory with mean velocity zero and whose extension increases upon increasing frequency. The explanation of these gaps and the observation of "quantized" velocity values as typical signatures of ratchet behavior can be found in [24] for the particle-like case and in [9] for the extended system. We have also verified that, when the soliton leaves the zone in which the array is contained, its motion becomes purely oscillatory, as expected [19].
Turning now to a more detailed comparison, we have to admit that the agreement with the collective coordinate theory of the previous section is not quite satisfactory. Figure $1 \mathrm{~b}$ makes this point clear by showing that neither the number of windows nor, of course, their locations, are correctly predicted, even in the simpler low frequency case. This situation is not changed in a relativistic calculation. Searching for an explanation of this problem, we analyzed in depth the simulations, finding out that a possible reason for this discrepancy is that the soliton shape changes during its motion along the inhomogeneities array (namely, its width is oscillating). This feature can not be accounted for within the framework of a one-coordinate treatment and therefore we set out to improve it in the next section.

\section{Improved collective coordinate theory}

In order to account for the phenomenology observed in the simulations, we resort to the generalized travelling wave ansatz for solving our problem, first proposed in $[25,26]$ for one and two degrees of freedom. As our starting point we rewrite equation (1) in the following way

$$
\dot{\phi}=\frac{\delta H}{\delta \psi}, \quad \dot{\psi}=-\frac{\delta H}{\delta \phi}-\beta \dot{\phi}-\sin (\phi) V(x)+f(t),
$$

where $\psi=\dot{\phi}$ and $H$ is the Hamiltonian for the unperturbed problem. Following the procedure as in [26] and assuming that $\phi$ has the form of the so-called Rice Ansatz [27]

$$
\begin{aligned}
\phi(x, t) & =\phi_{K}[x-X(t), l(t)] \\
& =4 \arctan \left(\exp \left[\frac{x-X(t)}{l(t)}\right]\right),
\end{aligned}
$$

where $l(t)$ is intended to account for the observed oscillations of the kink width, we find:

$$
\begin{aligned}
& M_{0} l_{0} \frac{\ddot{X}}{l}+\beta M_{0} l_{0} \frac{\dot{X}}{l}-M_{0} l_{0} \frac{\dot{X} \dot{l}}{l^{2}}=-\frac{\partial U}{\partial X}-q f(t), \\
& \alpha M_{0} l_{0} \frac{\ddot{l}}{l}+\beta \alpha M_{0} l_{0} \frac{\dot{l}}{l}+M_{0} l_{0} \frac{\dot{X}^{2}}{l^{2}}=K^{i n t}(l, \dot{l}, \dot{X})-\frac{\partial U}{\partial l},
\end{aligned}
$$

where $K^{i n t}(l, \dot{l}, \dot{X})=-\frac{\partial E}{\partial l}$ with

$$
E=\frac{1}{2} \frac{l_{0}}{l} M_{0} \dot{X}^{2}+\frac{1}{2} \frac{l_{0}}{l} \alpha M_{0} \dot{l}^{2}+\frac{1}{2} M_{0}\left(\frac{l_{0}}{l}+\frac{l}{l_{0}}\right),
$$

$M_{0}=8, l_{0}=1, \alpha=\pi^{2} / 12, q=2 \pi, \beta=1$ and $U(X, l)$ has the same form as $U(X)$ in equation (5) but with the denominators recast as $\cosh ^{2}\left[\left(X-x_{i}-n L\right) / l\right], i=1,2,3$.

In order to make more transparent the physical meaning of these equations, we can change variables by introducing the momentum $P(t)=M_{0} l_{0} X / l(t)$. Our equations become

$$
\begin{aligned}
\frac{d P}{d t}+\beta P & =-\frac{\partial U}{\partial X}-q f(t), \\
\alpha\left[\dot{l}^{2}-2 l \ddot{l}-2 \beta l \dot{l}\right] & =\frac{l^{2}}{l_{0}^{2}}\left[1+\frac{P^{2}}{M_{0}^{2}}\right]-1+\frac{2 l^{2}}{M_{0} l_{0}} \frac{\partial U}{\partial l} .
\end{aligned}
$$



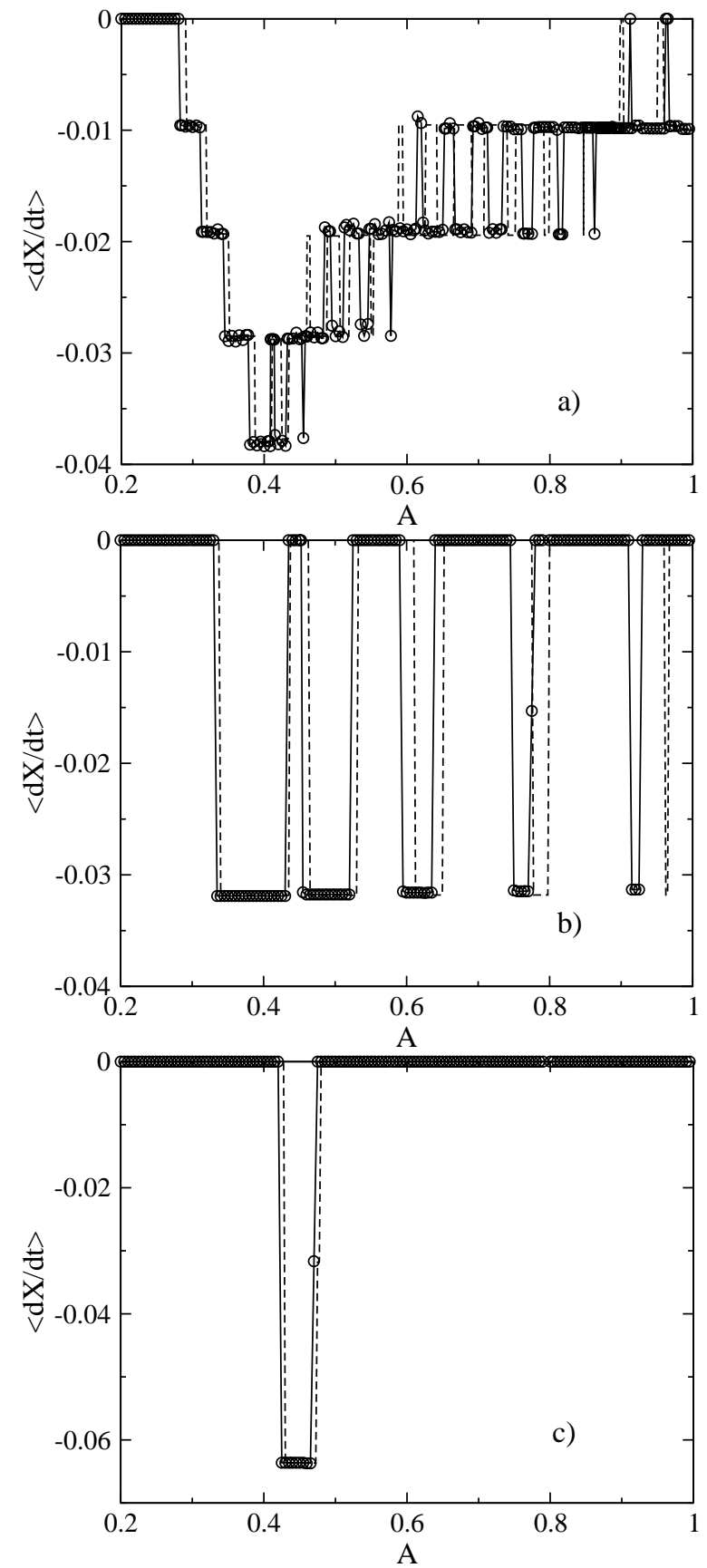

Fig. 2. Mean kink velocity $\langle d X / d t\rangle$ vs driving amplitude $A$ for different frequencies: a) $\omega=0.015$, b) $\omega=0.05$, c) $\omega=0.1$. Other parameters are the same as in Figure 1. Circles: direct numerical simulation of equation (1), the line being only a guide to the eye; dashed line: improved collective coordinate theory, equations $(11,12)$.

We thus see that indeed, in case the kink width oscillates, it necessarily couples to the translational motion, as the derivative of $U(X, l)$ in equation (11) contains $l$, whereas $l$ is in turn directly affected by the momentum. As we observe such oscillations in our simulations, our first approach with one degree of freedom must necessarily be incomplete.
The final step is to compare the improved theory to the simulations. Lacking analytical solutions, we have numerically solved the ordinary differential equations $(8,9)$, computing the kink velocity $\dot{X}(t)$ and its mean value. As shown by Figure 2, the comparison between our improved collective coordinate theory and the simulations is now excellent, as the window numbers are correctly estimated and their locations are very accurately predicted. We thus see that although the point particle approximation (collective coordinate $X(t)$ ) is enough to predict the appearance of a ratchet phenomenon, the correct description of the dynamics necessitates one additional degree of freedom, $l(t)$, arising from the fact that the fluxon, the "particle" in the ratchet, is an extended object that can show internal oscillations. Strikingly, in this way, the interplay of the two degrees of freedom leads eventually to a behavior truly indistinguishable from a point-particle ratchet.

\section{Conclusions}

In conclusion, we have proposed and tested in simulations an experimentally feasible and uncomplicated procedure to build a soliton ratchet using modified long Josephson junctions. The main advantage of this system is its simple design, that allows an easy implementation by means of indentations of the insulating layer (microshorts). An interesting feature of our system is its ability to rectify ac forces even if they are time-symmetric, something that cannot be accomplished by the homogeneous sG model $[6,7]$. We have been able to show analytically that the physical mechanism responsible for the appearance of the ratchet effect is the coupled dynamics of the center and width degrees of freedom, whose combined evolution is able to make fluxons (extended objects) behave as point-like particles.

An important issue about our results is whether or not they come from the fact that our system is deterministic or, in other words, whether or not they are robust and survive in the presence of noise. We have made several checks for different sets of parameters and we have verified that the sharp windows appearing in the deterministic case are simply smoothed due to the noise (for weak and intermediate noises). This smoothing affects the regions between windows as well, which become minima of the velocity instead of regions of zero velocity. Indeed, the scenario is very much as reported for point particles in [24], and the collective coordinate description remains basically correct. A careful characterization of the effects of noise is under way and will be reported elsewhere [28].

Finally, as regards the generality of the procedure presented here, we want to stress that our results open the door to many other applications. Indeed, the mechanism for the ratchet effect we have found, namely the coupling between translational motion and internal oscillations, will be relevant in general for topological solitons, such as those found in the $\phi^{4}$ and other nonlinear Klein-Gordon models. Such models describe applications in a variety of fields ranging from biophysics to pattern forming systems 
(see, e.g., [11,13] for references). Work along these lines, oriented specifically to assess the actual role of this phenomenon related to the macromolecules modelled by the $\phi^{4}$ equation (see [29] and references therein), is in progress.

This work has been supported by the Ministerio de Ciencia y Tecnología of Spain through grant BFM2000-0006 (AS), and by the International Research Training Group 'Nonequilibrium Phenomena and Phase Transitions in Complex Systems' (DFG, Germany).

\section{References}

1. J. Maddox, Nature 365, 203 (1993); J. Maddox, Nature 368, 287 (1994)

2. F. Jülicher, J. Prost, Rev. Mod. Phys. 69, 1269 (1997); P. Reimann, P. Hänggi, Appl. Phys. A 75, 169 (2002); P. Reimann, Phys. Rep. 361, 57 (2002)

3. H. Linke, W. Sheng, A. Löfgren, H. Xu, P. Omling, P.E. Lindelof, Science 286, 2314 (1999)

4. J.B. Majer, J. Peguiron, M. Grifoni, M. Tusveld, J.E. Mooij, Phys. Rev. Lett. 90, 05682 (2003)

5. F. Marchesoni, Phys. Rev. Lett. 77, 2364 (1996) 2364; A.V. Savin, G.P. Tsironis, A.V. Zolotaryuk, Phys. Lett. A 229, 279 (1997); Phys. Rev. E 56, 2457 (1997)

6. S. Flach, Y. Zolotaryuk, A.E. Miroshnichenko, M.V. Fistul, Phys. Rev. Lett. 88, 184101 (2002)

7. M. Salerno, Y. Zolotaryuk, Phys. Rev. E 65, 56603 (2002)

8. F. Falo, P.J. Martínez, J.J. Mazo, S. Cilla, Europhys. Lett. 45, 700 (1999)

9. F. Falo, P.J. Martínez, J.J. Mazo, T.P. Orlando, K. Segall, E. Trías, Appl. Phys. A 75, 263 (2002)

10. A. Sánchez, A.R. Bishop, F. Domínguez-Adame, Phys. Rev. E 49, 4603 (1994); B.Y. Zhu, L. Van Look, F. Marchesoni, V.V. Moshchalkov, F. Nori Physica E 18, 322
(2003); B.Y. Zhu, F. Marchesoni, F. Nori, Physica E 18, 318 (2003); F. Marchesoni, B.Y. Zhu, F. Nori, Physica A 325, 78 (2003)

11. A. Sánchez, A.R. Bishop, SIAM Review 40, 579 (1998)

12. D.W. McLaughlin, A.C. Scott, Phys. Rev. A 18, 1652 (1978)

13. Yu.S. Kivshar, B.A. Malomed, Rev. Mod. Phys. 61, 763 (1989)

14. Yu.S. Kivshar, Z. Fei, L. Vázquez, Phys. Rev. Lett. 67, 1177 (1991); Z. Fei, Yu.S. Kivshar, L. Vázquez, Phys. Rev. A 46, 5214 (1992)

15. Yu.S. Kivshar, A. Sánchez, O. Chubykalo, A.M. Kosevich, L. Vázquez, J. Phys. A: Math. Gen. 71, 5711 (1992)

16. S. Gredeskul, Yu.S. Kivshar, L.K. Maslov, A. Sánchez, L. Vázquez, Phys. Rev. A 45, 8867 (1992)

17. G. Carapella, G. Costabile, Phys. Rev. Lett. 87, 77002 (2001); E. Goldobin, A. Sterk, D. Koelle, Phys. Rev. E 63, 31111 (2001)

18. M. Salerno, N.R. Quintero, Phys. Rev. E 65, 025602 (2001)

19. N.R. Quintero, A. Sánchez, Phys. Lett. A 147, 161 (1998); N.R. Quintero, A. Sánchez, Eur. Phys. J. B 6, 133 (1998)

20. P. Jung, J. Kissner, P. Hänggi, Phys. Rev. Lett. 76, 1996 (1996); J.L. Mateos, Phys. Rev. Lett. 84, 258 (2000)

21. W.A. Strauss, L. Vázquez, J. Comp. Phys. 28, 271 (1978)

22. T.E. Dialynas, K. Lindenberg, G.P. Tsironis, Phys. Rev. E 56, 3976 (1997)

23. M.O. Magnasco, Phys. Rev. Lett. 71, 1477 (1993)

24. R. Bartussek, P. Hänggi, J.G. Kissner, Europhys. Lett. 28, 459 (1994)

25. F.G. Mertens, H.-J. Schnitzer, A.R. Bishop, Phys. Rev. B 56, 2510 (1997)

26. N.R. Quintero, A. Sánchez, F.G. Mertens, Phys. Rev. E 62, 5695 (2000)

27. M. Salerno, A.C. Scott, Phys. Rev. B 26, 2474 (1982); M.J. Rice, Phys. Rev. B 28, 3587 (1983)

28. L. Morales-Molina, F.G. Mertens, A. Sánchez, unpublished

29. M.V. Satarić, J.A. Tuszyński, Phys. Rev. E 67, 011901 (2003) 